\title{
Band-gap expansion in the surface-localized electronic structure of $\mathrm{MoS}_{2}(0002)$
}

\author{
Sang Wook Han, ${ }^{1}$ Gi-Beom Cha, ${ }^{2,}{ }^{*}$ Emmanouil Frantzeskakis, ${ }^{3}$ Ivy Razado-Colambo, ${ }^{3}$ José Avila, ${ }^{3}$ Young S. Park, ${ }^{4}$ \\ Daehyun Kim, ${ }^{5}$ Jihoon Hwang, ${ }^{5}$ Jeong Soo Kang, ${ }^{5}$ Sunmin Ryu,${ }^{6}$ Won Seok Yun,,${ }^{1,}$ \\ Soon Cheol Hong, ${ }^{1, \ddagger}$ and Maria C. Asensio $3, \S$ \\ ${ }^{1}$ Department of Physics and Energy Harvest-Storage Research Center, University of Ulsan, Ulsan 680-749, Korea \\ ${ }^{2}$ Dongnae School, San 7-1, Bugok-dong, Geumjeon-gu, Busan 609-719, Korea \\ ${ }^{3}$ Synchrotron SOLEIL, L'Orme des Merisiers, Saint Aubin-BP 48, 91192 Gif sur Yvette Cedex, France \\ ${ }^{4}$ Center for Superfunctional Materials, Department of Chemistry, Pohang University of Science and Technology, Pohang 790-784, Korea \\ ${ }^{5}$ Department of Physics, The Catholic University of Korea, Bucheon 420-743, Korea \\ ${ }^{6}$ Department of Applied Chemistry, Kyung Hee University, Yongin, Gyeonggi 446-701, Korea \\ (Received 5 January 2012; revised manuscript received 19 August 2012; published 6 September 2012)
}

\begin{abstract}
The electronic band structure of $\mathrm{MoS}_{2}$ single crystals has been investigated using angle-resolved photoelectron spectroscopy and first-principles calculations. The orbital symmetry and $k$ dispersion of these electronic states responsible for the direct and the indirect electronic band gaps have been unambiguously determined. By experimentally probing an increase of the electronic band gap, we conclude that a $\mathrm{MoS}_{2}(0002)$ surface localized state exists just below the valence band maximum at the $\Gamma$ point. This electronic state originates from the sulfur planes within the topmost layer. Our comprehensive study addresses the surface electronic structure of $\mathrm{MoS}_{2}$ and the role of van der Waals interlayer interactions.
\end{abstract}

DOI: 10.1103/PhysRevB.86.115105

PACS number(s): 78.66.Jg, 71.30.+h, 73.20.At, 79.60.Bm

\section{INTRODUCTION}

Layered materials constitute a rich and unexplored source of two-dimensional (2D) crystals with remarkable properties and potential applications in diverse fields of electronics, optics, energy, catalysis, and tribology. ${ }^{1,2}$ In particular, molybdenite $\left(\mathrm{MoS}_{2}\right)$, a substance available in enormous quantities in nature, is able to amplify electrical signals at room temperature by consuming much less power than traditional silicon and even graphene, which is widely considered as another remarkable emerging material in microelectronics. Most importantly, single-layer $\mathrm{MoS}_{2}(\mathrm{~S}-\mathrm{Mo}-\mathrm{S} ; \mathrm{1L})$ is a promising alternative for fabricating transistors from atomically thin 2D materials. ${ }^{3}$ Radisavljevic et al. ${ }^{4}$ have recently reported transistors with room-temperature mobility of the order of $200 \mathrm{~cm}^{2} / \mathrm{Vs}$, current on/off ratios of $1 \times 10^{8}$ and extremely low standby power dissipation. Hence quasi-2D MoS , very thin and easy to use in nanotechnology, has real practical benefits in the manufacture of nanoscale transistors, solar cells and light-emitting diodes (LEDs). The existence of an electronic band gap in $\mathrm{MoS}_{2}$ gives a notable superiority over alternative electronic materials without a natural gap in their band structure (e.g., graphene). For electronic devices, where engineers continue trying to make things smaller, lighter and faster, a semiconductor with an appropriate band gap is essential to turn transistors into on/off states, more efficiently than the current silicon-based technology.

However, the electronic properties of single or few-layer $\mathrm{MoS}_{2}$ are not still fully understood. While bulk $\mathrm{MoS}_{2}$ is a typical indirect-gap semiconductor with a band gap of $1.29 \mathrm{eV}^{5,6}$ a single-layer $\mathrm{MoS}_{2}$ behaves as a direct-gap semiconductor with a band gap of $\sim 1.8 \mathrm{eV}$. This evolution has been lately demonstrated using photoluminescence ${ }^{7,8}$ and Raman spectroscopy. ${ }^{9}$ Despite these remarkable findings, the causes and origin of this unpredictable behavior have not been so far understood. Even though the bulk electronic structure has been extensively examined over the last 40 years by theoretical and experimental studies, ${ }^{10-15}$ the effect of van der Waals (vdW) interactions on the indirect-to-direct gap transition as a function of the $\mathrm{MoS}_{2}$ thickness has not been directly observed. ${ }^{16-18}$

We demonstrate that a novel $\mathrm{MoS}_{2}$ (0002) surface state exists at the $\Gamma$ point, characterized by a larger (indirect) band gap than the bulk. This result clearly contrasts with the behavior of topological insulators, which are characterized by insulating bulk states and conducting surface states. ${ }^{19}$ Moreover, using angle-resolved photoelectron spectroscopy (ARPES), the selective light polarization cross section of the $\mathrm{MoS}_{2}$ valence band states has been used to put in evidence the orbital origin of the electronic states involved in the recently reported crossover gap transition. ${ }^{7,8}$ Our experimental findings together with first-principles calculations can provide a comprehensive description of the $\mathrm{vdW}$ driven transition from an indirect to a direct gap semiconductor in the isolated single-layer $\mathrm{MoS}_{2}$.

\section{EXPERIMENTAL AND THEORETICAL DETAILS}

ARPES is the most direct method for studying the electronic band structure of solids. Experiments were performed at the ANTARES beamline of SOLEIL. The energy and angle resolutions of the ARPES apparatus were better than $2 \mathrm{meV}$ and $0.1^{\circ}$. Experiments have been carried out using circular and linear polarized light in which the electric field vector lies perpendicular and parallel to the plane of incident light for $s$ and $p$-polarized photons, respectively.

In order to analyze the ARPES results, we have performed first-principles calculations using the full-potential linear augmented plane-wave method (FLAPW) ${ }^{20}$ implemented in the FLEUR code. ${ }^{21}$ The general gradient approximation (GGA) was used for the exchange-correlation potential. ${ }^{22}$ For the lattice parameter of films, we took as starting parameters the experimental bulk values $(a=3.160 \AA$ and $c=12.294 \AA) .{ }^{23}$ The $c$ 


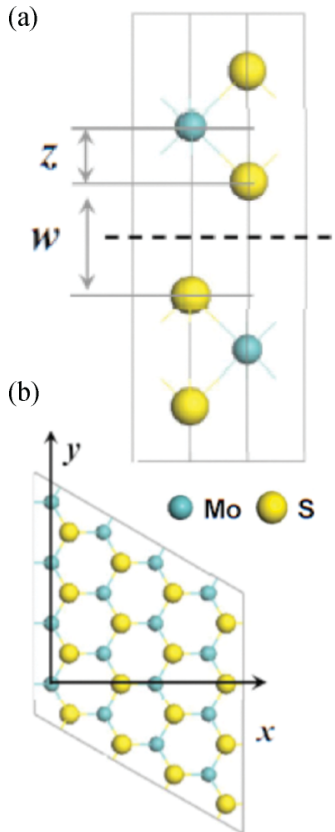

(c)

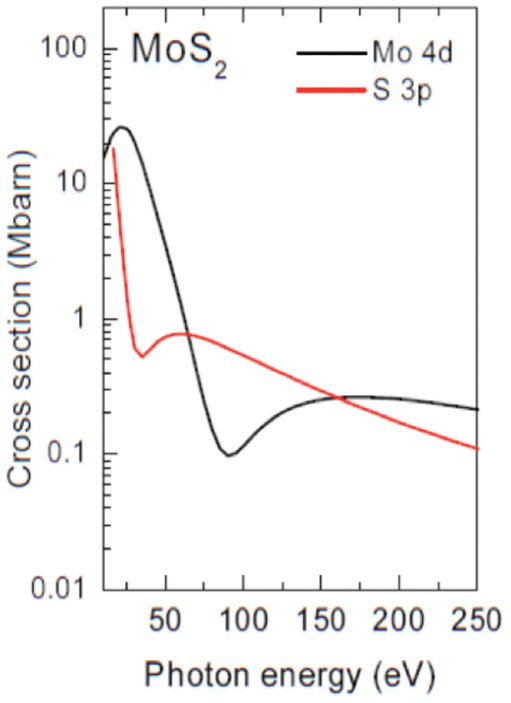

FIG. 1. (Color online) (a) Side view of the $2 \mathrm{H}-\mathrm{MoS}_{2}$ unit cell, containing two single layers. Dashed lines correspond to the (0002) plane. (b) Top view of the (0002) plane. (c) Theoretical atomic photoionization cross sections for the Mo $4 d$ and S $3 p$ subshells as a function of $h v$.

lattice parameter has been fully optimized for 6 layers $(\mathrm{L}) \mathrm{MoS}_{2}$ together with the internal atomic structural parameter $z$, which determines the S-S dimer separation, and the distance between layers $w$, i.e., vdW-bonding distance [see Fig. 1(a)]. The obtained values of $z=1.559 \AA$ and $w=3.132 \AA(c=12.509 \AA)$ increased by $\sim 4.7 \%$ and $\sim(5.1-5.3) \%$, respectively, in comparison to the bulk values of $z=1.49 \AA$ and $w=2.98 \AA$. Since variation of the lattice parameter $a$ has a significant effect on the band structure, ${ }^{18}$ it has been fixed at the aforementioned value. Energy cutoffs of 25 and $225 \mathrm{Ry}$ were employed for the basis expansion and the charge-potential within the interstitial region, respectively, and spherical harmonics with $l \geqslant 8$ inside the muffin-tin spheres. Brillouin zone integrations were performed on $15 \times 15$ Monkhorst-Pack grids. The surface region has been simulated using a single slab approximation. It is well known that local-density approximation (LDA) or GGA approaches fail to provide an accurate description of the vdW bonding in solids; LDA typically overbinds, whereas GGA typically underbinds such systems. ${ }^{24}$ Nevertheless, it should be noted that our calculations for $6 \mathrm{~L} \mathrm{MoS}_{2}$ yield an indirect band gap of $\sim 0.986 \mathrm{eV}$, which compares well with the experimental value of $\sim 1.38 \mathrm{eV},{ }^{8}$ thereby supporting our methodology.

Natural, single crystalline $\mathrm{MoS}_{2}$ (SPI, natural molybdenite, $4 \times 4 \mathrm{~mm}^{2}$ ) was cleaved in ambient air resulting in shiny flat surfaces and then introduced into the UHV chamber at a base pressure better than $5 \times 10^{-10}$ mbar. After annealing at approximately $700 \mathrm{~K}$ for more than 4 hours, the cleanliness and structural order were verified by the quality of the valence band dispersion and the constant energy (CE) contours obtained by an azimuthal angle scan. This annealing procedure is known to remove virtually all contamination from the surface without the formation of defects, while UHV cleaving may result in the formation of flakes on the surface. ${ }^{14,15}$ The most frequent allotrope of $\mathrm{MoS}_{2}$ is characterized by a $2 \mathrm{H}$ stacking. The $2 \mathrm{H}-\mathrm{MoS}_{2}$ unit cell [see Fig. 1(a)] extends over two S-Mo-S slabs, where the Mo atoms of one slab are placed on top of the $\mathrm{S}$ atoms of the other unit, and vice versa. The space group follows the hexagonal symmetry of $P 6_{3} / m m c\left(D_{6 \mathrm{~h}}^{4}\right)$, which consists of weakly vdW-bonded S-Mo-S slabs. Each slab contains two hexagonal planes of $\mathrm{S}$ atoms and an intermediate hexagonal layer of Mo atoms, the latter being trigonally prismatic coordinated to the six surrounding $\mathrm{S}$ atoms. Due to the quasi-2D nature of $2 \mathrm{H} \mathrm{MoS}_{2}$, peeling off the top surface layers is well known to produce a well-ordered $\mathrm{MoS}_{2}(0002)$ surface. ${ }^{25}$ This property enables the creation of a stable single layer of $\mathrm{MoS}_{2}$ by exfoliation. Due to the absence of inversion symmetry, single-layer $\mathrm{MoS}_{2}$ is classified to the $P 6 m 2\left(D_{3 \mathrm{~h}}^{1}\right)$ space group; a characteristic symmetry also observed for the $\mathrm{MoS}_{2}$ (0002) surface studied in the present work (see Sec. III).

\section{RESULTS AND DISCUSSION}

Figure 2 depicts the $k$-resolved electronic dispersion along the high-symmetry lines recorded with $h v=100$ and $120 \mathrm{eV}$ using two different polarizations. The high-symmetry points of the hexagonal surface Brillouin zone (SBZ) are plotted; Г/A: hexagon center, $\mathrm{K} / \mathrm{H}$ hexagon vertices and $\mathrm{M} / \mathrm{L}$ : middle of hexagon sides. The overall feature of the present band structure is quite consistent with previous ARPES studies. ${ }^{11-13}$ From one panel to another [see Fig. 2(a) versus Fig. 2(b)], the relative intensities change dramatically when linear polarization is turned from $p$ to $s$ type. Nevertheless, the binding energies of the photoemission features remain constant. According to the matrix elements effects, for $p$-polarized light, the intensity from out-of-plane orbitals is strongly enhanced. On the contrary, for $s$-polarized light, the intensity of the states associated to the in-plane orbitals is dominant. Consequently, we can attribute an out-of-plane (in-plane) character to the states located around $\Gamma(\mathrm{K})$.

On the other hand, some characteristic differences are observed at the slightly higher photon energy of $120 \mathrm{eV}$. In Fig. 2(c), the intensities between $2.5-4 \mathrm{eV}$ are enhanced in comparison to those of $100 \mathrm{eV}$, while they are diminished in the regions of $0.7-2.5$ and $4-6 \mathrm{eV}$ with a slight shift towards Fermi energy $\left(E_{\mathrm{F}}\right)$. In the case of $s$-polarized light, all features are similar to those observed with $100 \mathrm{eV}$, albeit with reduced intensities (data not shown). Moreover, in the left panel [see Fig. 2(a)], a chevron-shape dispersing band at the $\Gamma$ point is the most prominent feature with a binding energy of $\sim 1.1 \mathrm{eV}$. These facts indicate that bands at the $\Gamma / A$ point have a stronger $k_{\mathrm{z}}$ dependence, i.e., photon energy dependence, than those at the $\mathrm{M} / \mathrm{L}$ and $\mathrm{K} / \mathrm{H}$ points. This $k_{\mathrm{z}}$ dependence has been also revealed in a previous ARPES study. ${ }^{13}$

For reasons of clarity, ARPES features near $E_{\mathrm{F}}$ have been enhanced by using the new curvature method, an amelioration of the second derivative. ${ }^{26}$ In Figs. 3(a) and 3(b), a weak valence band maximum is located at $0.7 \mathrm{eV}$ just above the much brighter chevron-shaped dispersion which presents a maximum at a binding energy of $\sim 1.1 \mathrm{eV}$. Since the inelastic mean-free paths (IMFP) of the emitted electrons near $E_{\mathrm{F}}$ at $h v=70$ and $170 \mathrm{eV}$ are estimated to be $\sim 3.5$ and $\sim 6 \AA$, 

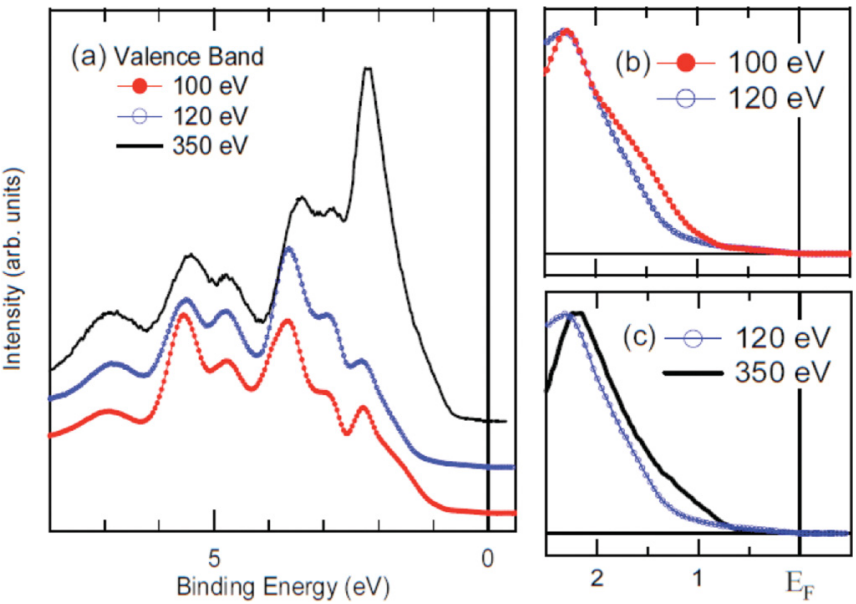

FIG. 5. (Color online) (a) Valence-band spectra of bulk $\mathrm{MoS}_{2}$ at three different photon energies after normalization at the binding energy of $\sim 6.5 \mathrm{eV}$. Comparison of each spectrum after normalization at the first peak below $E_{\mathrm{F}}$ : (b) $100 \mathrm{vs} 120 \mathrm{eV}$ and (c) $120 \mathrm{vs} 350 \mathrm{eV}$.

minimum at $\sim 3 \mathrm{eV}$. As a matter of fact, $\mathrm{CE}$ maps evidence a symmetry reduction where only $\Gamma \mathrm{K}$ is a mirror plane of the system. In other words, consecutive $\mathrm{K}$ points of the same Brillouin zone (BZ) are not equivalent. The corresponding space group is $P 6 m 2$ instead of the more symmetric $\mathrm{P}_{3} / \mathrm{mmc}$. This implies that the electronic structure of the $\mathrm{MoS}_{2}$ (0002) surface is rather similar to the exfoliated single layer, even though vdW interlayer interactions exist in the former.

Figure 5(a) shows the angle-integrated valence band spectra acquired with $h v=100,120$, and $350 \mathrm{eV}$. Every spectrum contains information obtained from both types of linear polarization. All peaks can be easily assigned from the calculated density of states (DOS) [see Fig. 6(c)]. The first strong peak acquired with $h v=350 \mathrm{eV}$, and having a binding energy of $\sim 2.2 \mathrm{eV}$, is dramatically reduced for lower photon energies because the latter are near the Cooper minimum of the Mo $4 d$ states [i.e., $h v=90 \mathrm{eV}$ and Fig. 1 (c)].$^{28}$ It is, therefore, clear that Mo $d_{\mathrm{z}}{ }^{2}$ orbitals are the origin of the strong intensity near $E_{\mathrm{F}}$.

Figure 5(b) presents a comparison of data acquired with $h v=100$ and $120 \mathrm{eV}$ after normalization at the first peak. Although spectra obtained at $120 \mathrm{eV}$ are further away from the Mo $4 d$ Cooper minimum, there is a stronger spectral weight at the low-energy side of the first peak, which is related to the chevron-shaped dispersion at $\Gamma$. This means that the chevron-shaped dispersing band at the $\Gamma$ mostly originates from out-of-plane $\mathrm{S} 3 p_{\mathrm{z}}$ orbitals, while the weak VBM is due to Mo $4 d_{\mathrm{z}}^{2}$ orbitals. Based on these facts, the bright spots of previous STM experiments on $\mathrm{MoS}_{2}$ must be the S, rather than the Mo atoms. ${ }^{29}$ Finally, Fig. 5(c) shows that spectral weight moves toward lower binding energies as the photon energy increases. This indicates that surface localized states may be characterized by a larger electronic band gap than the bulk.

In order to unravel the orbital origin of the VBM, as well as the characteristic band-gap expansion of the surface localized states, we have calculated the band structure of $6 \mathrm{~L} \mathrm{MoS}_{2}$ along the $\Gamma \mathrm{M}\left(1.148 \AA^{-1}\right)$ and $\Gamma \mathrm{K}\left(1.325 \AA^{-1}\right)$ high-symmetry lines of the hexagonal SBZ. The overall features of Fig. 6(a) are similar with previous calculations of the bulk. ${ }^{10-13}$ Colors and symbols denote the S $3 p$ and Mo $4 d$ orbitals. The size of the symbols indicates the degree of surface localization; i.e., the bigger symbol, the more localized is the state. The electronic band structure of $6 \mathrm{~L} \mathrm{MoS} 2$ presents an indirect gap $(\sim 1 \mathrm{eV})$ involving a VBM (set to zero energy) at $\Gamma$ and a conduction band minimum $(\mathrm{CBM})$ at about $0.55 \Gamma \mathrm{K}$. A direct band gap of (a) Band Structure of 6L

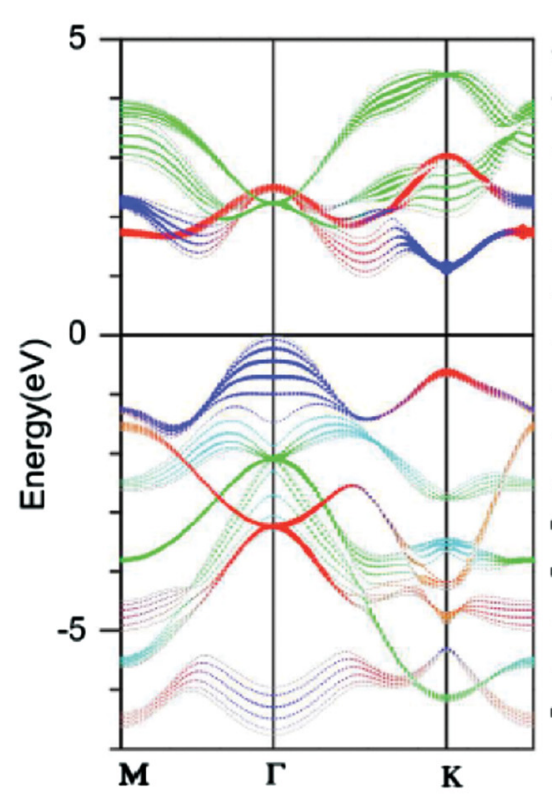

(b) $\Gamma$-point

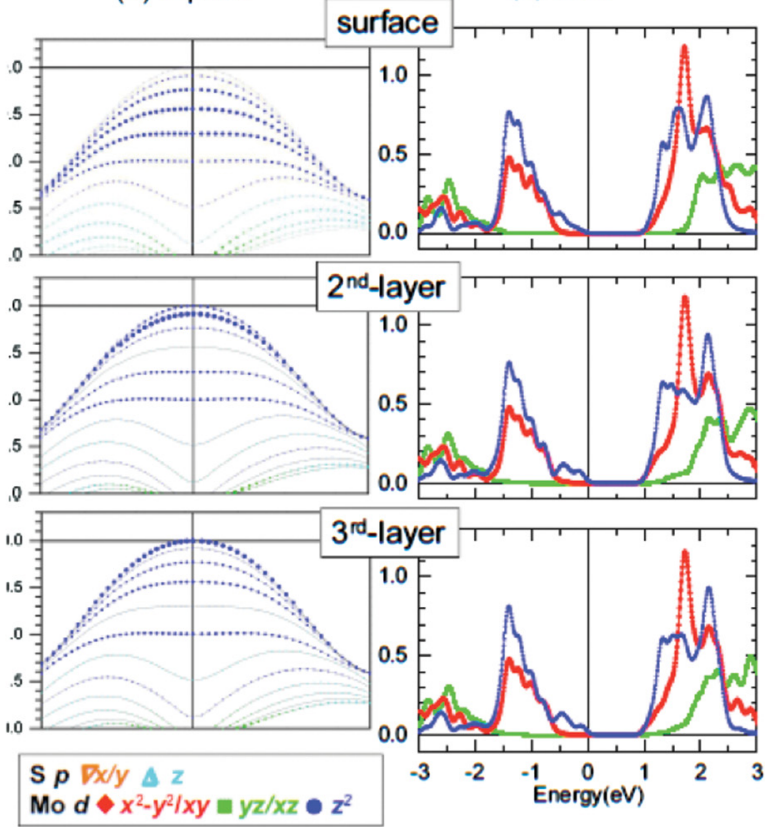

FIG. 6. (Color online) (a) Calculated band structure of $6 \mathrm{~L} \mathrm{MoS}_{2}$. The symbol size is proportional to the weight of the state within the surface. (b) Zoom on the states around the $\Gamma$ point. From top to bottom the symbol size indicates the weight of a state within the surface, the second layer, and the third layer, respectively. (c) Calculated density of states of Mo $d$ orbitals for $6 \mathrm{~L} \mathrm{MoS}_{2}$. 
$\sim 1.7 \mathrm{eV}$ lies at $\mathrm{K}$. The bands dispersing towards the $\mathrm{M}$ point mostly originate from out-of-plane Mo $d_{z}{ }^{2}$ orbitals, whereas those around $\mathrm{K}$ are induced by in-plane Mo $d_{x y}$ and $d_{x^{2}-y^{2}}$ orbitals. Both our experimental and theoretical data suggest that the VBM has a dominant Mo $4 d$ character, as compared to the states at slightly lower energy which mainly stem from $\mathrm{S} 3 p$ orbitals.

Drastic changes of band structure as a function of the number of layers are clearly observed at the VBM. The single state of $1 \mathrm{~L} \mathrm{MoS}_{2}$ is energetically split as the number of layers is increased. In the case of $6 \mathrm{~L} \mathrm{MoS}_{2}$, the state is split into six states [see Fig. 6(a)]. Detailed features are shown in Fig. 6(b), where the symbol size in the top, the middle, and the bottom panels was drawn so as to be proportional to the weight of a state within the surface, the second layer, and the third layer, respectively. Due to inversion symmetry, each layer from the surface to third layer is identical to the sixth, fifth, and fourth layers, respectively. As shown in Fig. 6(b), $d_{z}^{2}$ states (the third, fourth, and fifth states from the VBM) at the surface layer are split less, whereas the states of the third layer are significantly split so as to be located at the VBM. There is therefore a larger energy shift for surface localized states. This is in line with the out-of-plane character of the states at $\Gamma$ and due to the collapse of the vdW interlayer interaction in the limit of $1 \mathrm{~L}$ $\mathrm{MoS}_{2}$. This energy shift reveals the effect of vdW bonding in the electronic band structure of $\mathrm{MoS}_{2}$. On the other hand, as it should be expected, the in-plane orbitals responsible of the strong covalent intralayer bonding at the $\mathrm{K}$ point are almost independent of the thin film thickness. Moreover, the smaller band curvature of the uppermost surface localized states with respect to the bulk VBM explains why the effective mass increases upon lowering the number of layers. ${ }^{18}$ In line with the aforementioned findings, the calculated DOS manifests a clear thickness dependence [see Fig. 6(c)]. From the bottom to the top panels, the weights of weak $d_{z}^{2}$ orbitals at $-0.5 \mathrm{eV}$ below zero energy move towards higher binding energy, while the intensity of the peak at $1.5 \mathrm{eV}$ increases. The theoretical results strongly support our experimental findings suggesting that the surface localized states are accompanied by a larger electronic band gap than the bulk.

\section{CONCLUSIONS}

Using selective light polarization, our experimental results on $\mathrm{MoS}_{2}$ unambiguously identify the orbital character of the electronic states located around the $\Gamma$ and $\mathrm{K}$ points of the Brillouin zone. By a combined study of ARPES and first-principles calculations it has been possible to identify the existence of $\mathrm{MoS}_{2}$ (0002) surface localized states at $\Gamma$. These surface states originate from $S p_{\mathrm{z}}$ orbitals and they are characterized by a larger band gap than the bulk. Future studies might extend our findings to the surface states of different materials of the $2 \mathrm{H}-M X_{2}$ family $(M=\mathrm{Mo}, \mathrm{W} ; X=\mathrm{S}, \mathrm{Se}, \mathrm{Te})$.

\section{ACKNOWLEDGMENTS}

The authors acknowledge the valuable support services of the Synchrotron SOLEIL and in particular the precious help of Stephane Lorcy. This research was mainly funded by PHC STAR project No. 25852XH. This work was also supported by the NRF and MEST of Korea (Grant No. K2011-0020394). Moreover, part of the team has been subsidized by Grant Nos. 2009-0093818 and 2010-0008842. The work at the CUK was also financed by the NRF under Contract No. 2011-0022444.
*Present address: Dongnae Girls' School, San 7-1, Bugok-dong, Geumjeon-gu, Busan 609-719, Korea.

${ }^{\dagger}$ Present address: Graduate Institute of Ferrous Technology, Pohang University of Science and Technology, Pohang 790-784, Korea.

${ }^{\ddagger}$ schong@ulsan.ac.kr

§asensio@synchrotron-soleil.fr

${ }^{1}$ C. N. Rao et al., Angew. Chem., Int. Ed. 48, 7752 (2009).

${ }^{2}$ A. H. Castro-Neto and K. S. Novoselov, Rep. Prog. Phys. 74, 082501 (2011).

${ }^{3}$ K. S. Novoselov et al., Natl. Acad. Sci. USA 102, 10451 (2005).

${ }^{4}$ B. Radisavljevic et al., Nat. Nanotech. 6, 147 (2011); ACS Nano 5, 9934 (2011).

${ }^{5}$ K. K. Kam and B. A. Parkinson, J. Phys. Chem. 86, 463 (1982).

${ }^{6}$ S. C. Ray, J. Mater. Sci. Lett. 19, 803 (2000).

${ }^{7}$ A. Splendiani et al., Nano Lett. 10, 1271 (2010).

${ }^{8}$ K. F. Mak, C. Lee, J. Hone, J. Shan, and T. F. Heinz, Phys. Rev. Lett. 105, 136805 (2010).

${ }^{9}$ C. Lee et al., ACS Nano 4, 2695 (2010).

${ }^{10}$ L. F. Mattheiss, Phys. Rev. B 8, 3719 (1973).

${ }^{11}$ R. Coehoorn, C. Haas, J. Dijkstra, C. J. F. Flipse, R. A. de Groot, and A. Wold, Phys. Rev. B 35, 6195 (1987).

${ }^{12}$ K. Fives et al., J. Phys.: Condens. Matter 4, 5639 (1992).

${ }^{13}$ T. Böker, R. Severin, A. Muller, C. Janowitz, R. Manzke, D. Voss, P. Kruger, A. Mazur, and J. Pollmann, Phys. Rev. B 64, 235305 (2001).
${ }^{14}$ J. C. McMenamin and W. E. Spicer, Phys. Rev. B 16, 5474 (1977).

${ }^{15}$ J. R. Lince, S. V. Didziulis, and J. A. Yarmoff, Phys. Rev. B 43, 4641 (1991).

${ }^{16}$ T. S. Li and G. L. Galli, J. Phys. Chem. C 111, 16192 (2007).

${ }^{17}$ S. W. Han et al., Phys. Rev. B 84, 045409 (2011).

${ }^{18}$ W. S. Yun, S. W. Han, S. C. Hong, I. G. Kim, and J. D. Lee, Phys. Rev. B 85, 033305 (2012).

${ }^{19}$ D. Hsieh et al., Nature (London) 452, 970 (2008).

${ }^{20}$ E. Wimmer et al., Phys. Rev. B 24, 864 (1981); 26, 4571 (1982).

${ }^{21}$ http://www.flapw.de.

${ }^{22}$ J. P. Perdew, A. Ruzsinszky, G. I. Csonka, O. A. Vydrov, G. E. Scuseria, L. A. Constantin, X. Zhou, and K. Burke, Phys. Rev. Lett. 100, 136406 (2008).

${ }^{23}$ C. Wildervanck and F. Jellinek, Z. Anorg. Allg. Chem. 328, 309 (1964).

${ }^{24}$ M. Hasegawa and K. Nishidate, Phys. Rev. B 70, 205431 (2004).

${ }^{25}$ K. T. Park et al., J. Phys. Chem. 100, 10739 (1996); K. T. Park, M. Richards-Babb, J. S. Hess, J. Weiss, and K. Klier, Phys. Rev. B 54, 5471 (1996).

${ }^{26}$ P. Zhang et al., Rev. Sci. Instrum. 82, 043712 (2011).

${ }^{27}$ J. R. Lince et al., Surf. Sci. 210, 387 (1989).

${ }^{28}$ J. J. Yeh and I. Lindau, At. Data Nucl. Data Tables 32, 1 (1985).

${ }^{29}$ J. D. Fuhr, J. O. Sofo, and A. Saul, Phys. Rev. B 60, 8343 (1999). 